\title{
Panorama científico de la relación entre la violencia intrafamiliar y de género y la resiliencia familiar: posibilidades, retos y límites
}

\section{Scientific Overview of the Relation between Domestic and Gender Violence and Family Resilience: Possibilities, Challenges and Limits}

\author{
Ricardo A. Jaramillo-Moreno \\ ORCID: 0000-0002-7220-6978 \\ Carlos Alberto Cuevas Ramírez** \\ ORCID: 0000-0001-5481-6011 \\ Universidad Santo Tomás, Colombia \\ Recibido: 6 junio de 2019 \\ Revisado: 26 de agosto de 2019 \\ Aceptado: 4 de noviembre de 2019
}

\section{Resumen}

En el marco de la investigación titulada "Resiliencia en víctimas de violencia intrafamiliar y de género Fase 1", realizada por el programa de Psicología de la Universidad Santo Tomás de Aquino (Bogotá, Colombia), se realizó una revisión de literatura, que describió el estado del arte de las investigaciones y estudios reflexivos publicados entre los años de 2015 y 2019 con respecto a la resiliencia familiar frente a la violencia intrafamiliar y de género (VIFG). Se obtuvieron resultados preliminares de 3160 documentos, que fueron depurados a 74 artículos, que respondieron a criterios de inclusión como: Artículos Originales, de Reflexión, Revisión de literatura, Estudios Cualitativos, Cuantitativos o Mixtos publicados entre 2015 y 2019. La búsqueda se realizó en las bases de datos: PubMed, Ebsco Host, Science Direct, Scopus, PsyıNFo, SAGE, Sprinter, y Taylor \& Francis. Se encontró una tendencia de estudios aplicados, cualitativos, descriptivos con población participante femenina, infantil y adolescente con reporte de violencia intrafamiliar, realizados en Estados Unidos, Reino Unido

Artículo de investigación. Citar como: Jaramillo-Moreno, R. A. \& Cuevas, R. C. A. (2020). Panorama científico de la relación entre la violencia intrafamiliar y de género y la resiliencia familiar: posibilidades, retos y límites. Diversitas: Perspectivas en Psicología, 16(1), 113-130. Dol: https://doi.org/10.15332/22563067.5544

Grupo de Investigación psicología, familia y redes. Producto del proyecto titulado: Resiliencia en víctimas de Violencia intrafamiliar y de Género Fase 1, realizada por el Programa de Psicología de la Universidad Santo Tomás, Bogotá, Colombia; Fodein N. ${ }^{\circ} 18650020$

Autor de correspondencia: Ricardo Jaramillo. Correo electrónico: ricardojaramillo@usantotomas.edu.co. Dirección postal; Facultad de Psicología, Universidad Santo Tomás, Sede Campus, San Alberto Magno. Autopista Norte, Calle 209, Kilómetro 1,6, Cl, Bogotá, Cundinamarca. 
y Canadá. Sin embargo, se pudo observar que la literatura hace referencia indirecta a la resiliencia familiar, pues en mayor medida se refiere al afrontamiento, recuperación y factores protectores, como fenómenos de interés relacionados con la VIFG. Se concluye que aún son pocos los estudios en los que se incluya explícitamente el fenómeno del género en la vIF, que describen la emergencia de la resiliencia familiar y alternativas de intervención para su desarrollo.

Palabras clave: resiliencia; resiliencia familiar; violencia intrafamiliar; violencia de pareja; género.

\section{Abstract}

In the frame of the first phase of the research titled "Resilience in Victims of Domestic and Gender Based Violence", carried out by the Psychology academic program at the Santo Tomas University (Bogota, Colombia), a literature review was carried out which described the State of the Art of studies published between 2015 and 2019 regarding Family Resilience against Domestic and Gender-based Violence. There were preliminary results gathered from 3160 documents, which were later reduced to 74 articles according to the following criteria: original articles, rational thinking, literature review, and qualitative data, quantitative data, or mixed methods research published between 2015 and 2019. The research was carried out using the following Databases: PubMed, Ebsco Host, Science Direct, Scopus, PsyINFO, SAGE, Sprinter, and Taylor \& Francis. A particular trend has been identified as a result of the research, with a large set of studies using mostly applied, qualitative and descriptive data of female, child and teenager participants who have reported cases of domestic violence, conducted in the United States, the United Kingdom, and Canada. However, it was observed that the literature makes indirect reference to family resilience, since for the most part it refers to coping mechanisms, recovery, and protective factors as phenomena of interest related to Domestic and Gender-based Violence. It is concluded that there are still very few studies which, while describing the emergence of family resilience and intervention alternatives for its development, explicitly include a gender approach or gender-related elements in cases of domestic violence.

Keywords: resilience; Family Resilience; Domestic Violence; Partner Violence; Gender.

\section{Introducción}

Dentro de los tipos de violencia, la Organización Mundial de la Salud (World Health Organization [wHo], 2014), destaca que la violencia interpersonal es la: que "ocurre entre miembros de una familia, en la pareja, entre amigos, conocidos y desconocidos, y que incluye el maltrato infantil, la violencia juvenil, la violencia en la pareja, la violencia sexual y el maltrato de las personas mayores" (wHo, p. viii). Al respecto, en esta investigación se tiende la violencia intrafamiliar (VIF) o violencia doméstica cómo el complejo relacional donde todos los miembros de la familia se encuentran implicados en una pauta de violencia que se ejerce en distintos modos jerárquicos y con múltiples manifestaciones, que van desde insultos, hasta descalificaciones severas y agresiones físicas (Brino \& Souza, 2016).

Desafortunadamente cada vez más estudios e investigaciones reportan reiterativamente el abordaje de este fenómeno, dado que su prevalencia permite estimar que se ha transformado en un problema para distintas disciplinas e instituciones que han aproximado su quehacer a esta dinámica en crecimiento a nivel mundial. Al respecto, la denominada onu- Mujeres (2017) refiere a que alrededor del mundo cerca del $35 \%$ de las mujeres han sido víctimas de la violencia y acoso. Sin embargo, y 
en otras proporciones, se presenta un subregistro debido a la no denuncia de violencia, de abuso y violencia sexual. Lo anterior, preocupantemente es propiciado en buena medida por su compañero sentimental. Así pues, se "estima que de las 87,000 mujeres que fueron asesinadas globalmente en el 2017, más de la mitad ( 50,000 -58 por ciento) fueron asesinadas por sus parejas o miembros familiares." (United Nations Office on Drugs and Crime, 2018, citado por onu-Mujeres, 2017). En Colombia, se encuentra que en 2018 de 65.736 casos reportados, 50.120 corresponden a mujeres y 15.616 a hombres (Instituto Nacional de Medicina Legal, 2018).

Regresando sobre los miembros de la familia que experimentan la violencia, es de notar que los perpetradores, usualmente las parejas, han sido víctimas de la violencia en la infancia, patrón que repiten con sus parejas en la vida adulta. (ONU Mujeres, 2017). Así pues, para una comprensión amplia acerca de esta noción intergeneracional, vale la pena recordar la definición de familia que se ha construido desde el modelo solidario desarrollado por el Instituto Colombiano de Bienestar Familiar (ICBF), en el cual se consignan las aproximaciones relacionales a este sistema. La familia se define como una unidad que depende de otros sistemas, es decir, es ecosistémica por la interconexión que se establece entre los miembros al interior de la misma como por las relaciones con las instituciones que la circundan; es un centro de construcción de solidaridades y de destino que derivan de la emergencia de los vínculos entre los distintos actores para favorecer la autonomía, la idiosincrasia social de la familia y los modos en los cuales la subjetividad y los mundos relacionales se interpelan para actuar como coevoluciones de los miembros implicados en cada sistema (ICBF, 2006)

Con esto, se asume que la familia va más allá de la consanguinidad, puesto que las familias actuales no solo se definen por rasgos filogenéticos, sino ontológicos, remitiendo a una noción de la naturaleza de estas relaciones enmarcadas en las necesidades que suple, y a la elección de permanencia o ausencia que los miembros de esta configuración asumen. Así mismo, la familia facilita además aspectos de la construcción política de los sujetos, centrando también las responsabilidades frente a las instituciones gubernamentales y de estado para la inclusión de políticas públicas (ICBF, 2006).

Ahora, si se establece una aproximación a la familia en este orden, no es posible dejar de entender que la emergencia de la pauta violenta de este sistema tiene repercusiones relacionales que devienen de algunos principios como la equifinalidad y la equicausalidad, como reguladores de los inicios y los finales de unos comportamientos violentos. Es decir, desde esta aproximación se espera que se realicen lecturas específicas de cada familia ya que las mismas causas pueden devenir en finales diferentes y finales similares devienen de causas diferentes. Finalmente, comprender este fenómeno desde la visión sistémica favorece la lectura en las relaciones, lo que amplía el espectro, para dejar de ver sólo un responsable de esta violencia, entendiendo cómo se han gestado las interacciones y de qué modo todos los miembros participan de este proceso violento familiar (Hernández \& Bravo, 2004).

En conexión con esta comprensión relacional, es imposible omitir el enfoque de género como herramienta para comprender las puntuaciones que se suscitan entre los géneros (Cuevas, 2017). Así pues, se puede comprender de qué modo esta participación se enmarca en lógicas patriarcales, heterosexistas, sexistas y de clase, en donde, la noción de diferencia de los géneros y de los estereotipos que la atraviesan implican validaciones de la violencia que emerge en un contexto familiar. Se cree que el hombre tiene derechos sobre la mujer, que los hijos varones deben dar ejemplo y liderar el sistema fraternal, que las hijas deben ser protegidas y estas creencias que se enmarcan en el género al ser abordadas en procesos investigativos facilitarán las transformaciones sociales necesarias para la consecución de contextos más igualitarios y equitativos.

\section{Violencia intrafamiliar, género y resiliencia}

Ante el impacto que representa la VIFG, y considerando la necesidad de dar respuesta al fenómeno y a la posición de género, es valioso considerar acciones, intervenciones y estudios que no solamente visibilicen la problemática, también las alternativas para su abordaje. Esto es, desde la salud, la salud 
mental positiva, el crecimiento, la generatividad y la transformación, o en otras palabras la "resiliencia" (Cyrulnik, 2010; Rutter, 1993) y aquí la "resiliencia familiar" (Kotliarenco \& Gomez, 2010; Walsh, 2011) lo que unido a la participación, igualdad y empoderamiento, estos últimos relacionados con los objetivos del milenio de la onu, permitan dar soluciones a esta situación (Jahoda, 1958; JaramilloMoreno, 2017; Jaramillo-Moreno \& Cuervo, 2016, Lluch, 2002, onu Mujeres, 2017).

De lo anterior, la resiliencia se comprende como la capacidad de protegerse, afrontar y salir transformado generativamente de situaciones de vulnerabilidad, adversas, traumáticas, disruptivas o que representen gran exigencia para las personas (Cyrulnik, 2010, Rutter, 1993, Werner \& Smith, 1982). Esta característica puede ser observada a través de competencias o recursos cognitivos, solución de problemas, resignificación de eventos, flexibilidad psicológica, estados afectivos, afrontar y trascender sentimientos intensos, ecuanimidad, sensación de ser amado y valorado, y comportamentales como la toma rápida de decisiones, la generación de redes, entre otras (Mrazek \& Mrazek, 1987).

Ahora, este fenómeno de resiliencia no se da de forma aislada, pues lo consideramos relacional y emergente, es decir, que tiene su base en las relaciones y la identidad familiar (Jaramillo-Moreno \& Cuevas, 2018; Walsh, 2004), pero no se presenta únicamente a partir de la confrontación de la familia con la adversidad, sino que responde a una construcción identitaria previa, a las relaciones entre la familia y los sistemas amplios (Imer-Black, 2000) y que puede ser comprendido en forma de factores protectores. Estos factores funcionan como dispositivos cambiantes y adaptativos, es decir, se adaptan ante diferentes situaciones, sean estas de riesgo, transformación, construcción, o cómo aquí, ante la adversidad en forma de resiliencia familiar (Jaramillo-Moreno \& Cuervo, 2016; JaramilloMoreno\& Cuevas, 2018).

Hasta aquí, retomando la necesidad de visibilizar el fenómeno de la resiliencia familiar; la prevalencia e impacto de la VIFG, incluso desde varias disciplinas, no es del todo claro desde el panorama investigativo, explicativo y de intervención reciente. No resulta claro cómo ante este acontecimiento de violencia, las familias, los niños y los adolescentes, se protegen, salen fortalecidos, no replican los patrones de violencia modelados históricamente, o como complemento no permiten los síntomas de agresión física y depresión en adolescentes (Kassis, Artz, Scambor, Scambor, \& Moldenhauer, 2013). Así, esta resiliencia familiar tiene el papel mediador en la familia y facilita el control de la ira en la relación entre las dimensiones de los patrones de comunicación familiar y la tendencia a la violencia (Hashemi \& Akbari, 2017).

El otro mecanismo es a través de la construcción de factores personales y culturales (Wortham, 2014) la conexión con la cultura y las tradiciones ancestrales y la acogida de la comunidad (Inman \& Rao, 2018; Roy, 2018) favorecen la resistencia, la transformación y el empoderamiento de las personas a pesar de la situación de violencia por la que atraviesan, o cómo fueron relevantes para la recuperación de los efectos que produce la violencia doméstica. Ante esta situación nos planteamos la siguiente pregunta: ¿cuál es estado del arte frente al fenómeno de la resiliencia familiar y su relación con la violencia intrafamiliar y de género entre 2015 y 2019 ?

\section{Método}

Se realizó una revisión de la literatura desde la metodología del Estado del Arte, la cual para Grant y Booth (2009) se caracteriza por ser un subtipo de la metodología de revisión de literatura, pero tiende a abarcar más tópicos, dado que puede aportar más perspectivas acerca de un problema o fenómeno o evidenciar un área que seguramente puede necesitar mayores investigaciones.

Se tuvieron en cuenta publicaciones disponibles en bases de datos abiertas o por suscripción, que hayan sido publicados entre 2015 y 2019.

Se revisaron los siguientes tipos de publicación: artículos originales, reflexiones teóricas, y artículos de revisión. De diferentes metodologías, es decir, tipos de investigación cualitativa, cuantitativa o mixta, y que fueran realizados en cualquier lugar de procedencia. Las palabras clave utilizadas fueron, en español: "Resiliencia", "Resiliencia Familiar", "Violencia Intrafamiliar" y "género", 
y en inglés; "Family Resilience", "Domestic Violence" y "Gender".

La recolección y análisis de datos se realizó determinando las categorías previas primarias y secundarias. Las primarias obedecieron al objetivo de la revisión, es decir, "Resiliencia familiar", "Violencia Intrafamiliar", "Programas y estrategias de promoción de resiliencia”. Dentro de la categoría primaria de resiliencia familiar, se tuvieron las siguientes subcategorías: "Factores Promotores", "Recursos Familiares Resilientes" y "Programas y estrategias de promoción de resiliencia " $y$, dentro de violencia intrafamiliar las subcategorías fueron: "VIF y de Género VIFG", "Adultos con historial o sobrevivientes a la VIFG" y "VIFG niños, niñas y adolescentes".

Las categorías secundarias respondían a la identificación y caracterización de los artículos, estas categorías fueron: año, nombre de la publicación, país de procedencia, tipo de estudio, diseño de investigación, tipo de artículo, población, tipos de violencia asociados con la VIF, género, resiliencia, factores protectores, afrontamiento, crecimiento post traumático, recuperación e intervención, disciplinas relacionadas con el estudio.

\section{Procedimiento}

Durante la primera fase se realizó la búsqueda preliminar e identificación de los artículos, se consideró la ventana de observación expuesta anteriormente, para la primera depuración. Posteriormente se realizó un segundo filtro, relacionado con las categorías previas y obtener una base de datos consolidada. Luego, se descargaron los artículos y organizados en el Gestor de Refencias Ref Works ${ }^{\circledR}$. Posterior a esto, se procedió a la creación de una base de datos (Excel $($ ) para realizar la descripción de las categorías secundarias. Frente a las categorías primarias se realizó una codificación teórica (Flick, 2004) en la que se identificaron los temas emergentes en los resultados y conclusiones de los artículos. Después, se clasificaron en las categorías primarias (Taylor \& Bogdan, 1987) y finalmente se realizó la codificación abierta en el que se elaboraron notas que revelaran hallazgos, para su posterior ubicación en códigos o categorías y subcategorías, que permitieran la comprensión de los textos y a su vez facilitaran la generación de conclusiones o reflexiones para la discusión. Este análisis categorial planteó una última depuración de los documentos y posterior interpretación y discusión de los resultados.

\section{Resultados}

En la primera revisión se encontraron 3160 documentos, dentro de los cuales se encontraban ponencias, memorias de congresos, capítulos de libros, libros, cartas al editor, artículos originales (empíri$\cos )$, de reflexión y artículos de revisión.

De esta primera fase y utilizando los criterios de inclusión, se disminuyó la muestra de documentos a 80. Posteriormente frente a la construcción de la base de datos y la lectura reflexiva se excluyeron de la revisión cuatro (4), debido a que no se observó una relación directa con las categorías de análisis principales, y debido a que algunos de los documentos estaban incompletos, no entraban con exactitud en la ventana de observación 2015-2019, o hacían referencia a libros o capítulos de libro. Posterior a esta última revisión se realizó el análisis de 74 artículos.

Considerando las categorías secundarias se observó que el año de mayor publicación fue el de 2018, con 20 artículos, así mismo se observa como desde el 2015 hasta el 2019, considerando este último la cantidad de publicaciones, independientemente de su tipo han ido incrementando.

Tabla 1.

Artículos publicados entre los años 2015 - 2019

\begin{tabular}{cc}
\hline Año & Artículos \\
\hline 2015 & 13 \\
2016 & 12 \\
2017 & 18 \\
2018 & 20 \\
2019 & 11 \\
\hline Total & 74 \\
\hline
\end{tabular}

Fuente: elaboración propia.

De acuerdo con los países de procedencia el mayor número de publicaciones se encuentran: Estados Unidos, Reino Unido, Canadá, Australia, Sudáfrica, 
Uganda, España y Turquía, los restantes con una publicación, se encuentran distribuidos en diferentes países (Tabla 2).

Tabla 2.

País de Procedencia de los artículos

\begin{tabular}{cc}
\hline País de Procedencia & Frecuencia \\
\hline Estados Unidos & 29 \\
Reino Unido & 14 \\
Canadá & 7 \\
Sudáfrica & 3 \\
Australia & 3 \\
Turquía & 2 \\
Uganda & 2 \\
España & 2
\end{tabular}

Fuente: elaboración propia.

Considerando las publicaciones que visibilizan los fenómenos de la VIFG, resiliencia y otros fenómenos asociados (Tabla 3), se observa la predominancia de artículos originales donde se socializan resultados de Investigación y con menor frecuencia los de revisión.

Tabla 3.

Tipos de artículo publicados

\begin{tabular}{lc}
\hline Tipos de Artículo & Frecuencia \\
\hline Artículo original & 58 \\
Artículo reflexivo & 1 \\
Artículo de revisión & 15 \\
\hline
\end{tabular}

Fuente: elaboración propia.

También se destaca una gran diversidad de publicaciones (Tabla 4), las que mayor difusión hacen del tema son: el Journal Of interpersonal Violence, Child Abuse \& Neglect, Journal of Family Violence, Child and Adolescent Social Work Journal, Children and youth services review, Journal of Child \& Adolescent Trauma, Trauma, Violence, \& Abuse y Aggression and Violent Behavior.
Tabla 4.

Revistas que publican acerca de resiliencia violencia intrafamiliar y de pareja entre 2015 - 2019

\begin{tabular}{lc}
\hline Revistas & Cant Artículos \\
\hline Journal of Interpersonal Violence & 13 \\
Child Abuse \& Neglect & 8 \\
Journal of Family Violence & 5 \\
$\begin{array}{l}\text { Child and Adolescent Social Work } \\
\text { Journal }\end{array}$ & 3 \\
Children and youth services review & 3 \\
Journal of Child \& Adolescent & 3 \\
Trauma & 3 \\
Trauma, Violence, \& Abuse & 2 \\
Aggression and Violent Behavior & \\
\hline
\end{tabular}

Fuente: elaboración propia.

De acuerdo con los tipos de investigación, incluyendo los artículos de revisión de literatura (Tabla 5), se desarrollan desde perspectivas cualitativas, cuantitativas y mixtas, aunque la VIFG y resiliencia, tienden a ser estudiadas desde posturas cualitativas.

Tabla 5.

Tipos de estudios publicados por las revistas

\begin{tabular}{cc}
\hline Categoría & Frecuencia \\
\hline Cuantitativo & 27 \\
Cualitativo & 43 \\
Mixto & 4 \\
\hline Total & 74 \\
\hline
\end{tabular}

Fuente: elaboración propia.

En complemento, las publicaciones reportadas respondían a diseños de investigación diversos, aunque los más frecuentes fueron los descriptivos (cualitativa, cuantitativa y mixtos); cualitativamente se observan diseños de tipo narrativo, fenomenológico u otros, al respecto de estos últimos, se observaron sistematizaciones de experiencias o enfoques afines, y menos frecuentes son estudios de tipo experimental, etnográficos y teoría fundamentada (Tabla 6). 
Tabla 6.

Diseños utilizados en investigaciones cualitativas, cuantitativas o mixtas frente al estudio de la resiliencia y la VIFG.

\begin{tabular}{lc}
\hline Categoría & Frecuencia \\
\hline Narrativo & 5 \\
Descriptivo & 58 \\
Multivariado & 2 \\
Fenomenológico & 3 \\
Etnográfico & 1 \\
Teoría fundamentada & 1 \\
Pre-cuasi Experimental & 2 \\
Otro & 2 \\
\hline Total & 74 \\
\hline
\end{tabular}

Fuente: elaboración propia.

Los participantes o grupos de interés que refirieron investigaciones obedecieron a grupos por género $y$ ciclo vital, de esta forma se observaron predominantemente estudios de mujeres, niños y adolescentes, seguidos por poblaciones de grupos culturales; menos frecuente fueron los estudios con adultos, toda vez que estos referían a investigaciones en las que las participantes también eran mujeres y casi no participaron hombres, salvo aquellos que participaron en estudios de tipo retrospectivo, es decir, con historial de VIF en su infancia (Tabla, 7).

Tabla 7.

Poblaciones objeto de investigación en resiliencia y violencia intrafamiliar

\begin{tabular}{lc}
\hline Categoría & Frecuencia \\
\hline Familias & 4 \\
Niños & 18 \\
Mujeres & 27 \\
Europeos & 1 \\
Pueblos Ancestrales & 3 \\
Adolescentes & 17 \\
Adultos & 4 \\
\hline Total & 74 \\
\hline
\end{tabular}

Fuente: elaboración propia.
En complemento de los grupos de interés, se observó la distinción de los estudios según los tipos de violencia en la familia, a pesar de la utilización de las palabras clave durante la búsqueda (Tabla 8). Así pues, se observa una mayor cantidad de estudios orientados a la violencia intrafamiliar (Domestic Violence) y violencia de pareja (Intimate Partner Violence). Otros estudios destacan que la presencia de otros tipos de violencia o acontecimientos adversos en los cuales la violencia intrafamiliar emerge como consecuencia. En este mismo sentido, se observaron estudios donde la violencia política explícitamente sirve de contexto para la emergencia de la violencia intrafamiliar, Por último, se observó que sólo un estudio hacía mención explícita a la violencia intrafamiliar en el marco del género.

Tabla 8.

Tipos de violencia relacionados con la resiliencia familiar y el género.

\begin{tabular}{lc}
\hline Categoría & Frecuencia \\
\hline Violencia de pareja & 27 \\
Violencia intrafamiliar & 36 \\
Violencia de género & 1 \\
Violencia política & 1 \\
Varios tipos de violencia & 9 \\
\hline Total & 74 \\
\hline
\end{tabular}

Fuente: elaboración propia.

Se observó en cuanto a la relación violencia intrafamiliar y resiliencia (Tabla 9), que no siempre los estudios hacen referencia a esta última, sino que se observa como una categoría implícita, pues emergería ante la situación violenta o a veces de forma intencional por parte de los investigadores. Esto es, como programas que promueven su desarrollo, de esta manera se observó que los estudios explícitamente se refieren a la resiliencia en una baja proporción, mientras que sería una variable paralela o secundaria vinculada con el afrontamiento de la VIFG, la recuperación, el crecimiento postraumático o comprendida como un factor protector. 
Tabla 9.

Relación entre VIF y resiliencia.

\begin{tabular}{lc}
\hline Categoría & $\%$ \\
Resiliencia & 34.83 \\
Afrontamiento & 28.09 \\
Recuperación & 16.85 \\
Factores protectores & 14.61 \\
Crecimiento postraumático & 5.618 \\
\hline Porcentaje acumulado & 65.17 \\
\hline
\end{tabular}

Fuente: elaboración propia.

En cuanto al interés disciplinar (Tabla 10), se observó que la psicología y los estudios interdisciplinares, son los principalmente interesados en la relación entre VIFG y RF, seg uidos por el Trabajo Social y las Ciencias de la Salud, es decir, se observó una postura interdisciplinar, pero en la salud, y diferenciada de la enfermería; y por último en la Educación.

Tabla 10.

Disciplinas que refieren estudios relacionados con la VIFG Y RF.

\begin{tabular}{lc}
\hline Disciplina & Número de Artículos \\
\hline Psicología & 19 \\
Sociología & 1 \\
Trabajo social & 15 \\
Terapia ocupacional & 0 \\
Medicina & 0 \\
Enfermería & 1 \\
Otras Ciencias de la Salud & 12 \\
Educación & 1 \\
Interdisciplinariedad & 23 \\
Otras & 2 \\
\hline Total & 74 \\
\hline
\end{tabular}

Fuente: elaboración propia.

De acuerdo con el análisis cualitativo de los documentos podemos afirmar que la resiliencia familiar se puede comprender como un continuo, en el sentido de que existen factores que la promueven, o lo que sería lo mismo, que son protectores de la violencia, y que facilitan su emergencia. Por otro lado, se re- conocen los recursos resilientes o la resiliencia mediante los cuales las personas afrontan la adversidad, aprenden de ella y se transforman. Así mismo, la literatura encontrada, si bien destaca la emergencia del fenómeno, las Ciencias Sociales y de la Salud, han empezado a desarrollar programas que facilitan o propenden por el desarrollo de estos recursos ante diferentes situaciones, aquí, particularmente la vIFG.

En cuanto a los factores que promueven estos fenómenos de violencia, podemos destacar los provenientes de las dinámicas, identidad e historia familiar, y que pueden ser comprendidos como la promoción de la regulación emocional y el autocontrol (Cassé et al., 2018; Yule, Houston, \& Grych, 2019), esto como parte de la crianza (Arslan, 2016), el apego seguro, (Bain \& Durbach, 2018, Bowen, 2015) el apoyo al interior de la familia nuclear (Cameranesi \& Piotrowski, 2018) y de la familia extensa (Dhunna, Lawton, \& Cram, 2018) el modelamiento de actitudes y acciones como la perseverancia, la fe, la empatía y el establecimiento de hogares estables (Stephens \& Aparicio, 2017).

A nivel meso y exo sistémico, la identidad cultural entendida como los factores culturales, las prácticas culturales y costumbres (Walters, 2016) la identidad bicultural (Sirikantraporn, 2017) el modelamiento de estilos no violentos y la recuperación de la voz de los ancianos (Asay, DeFrain, Metzger, \& Moyer 2016, Burnette, 2018), el papel positivo de la religión y la espiritualidad (Ghafournia, 2017; Schaefer, Howell, Thurston, Kaufman, 2018; Smith, Lenz, \& Strohmer, 2017) y el acceso a redes de apoyo paralelas a la familia (Schofield, Biggart, Ward, \& Larsson, 2015).

A nivel exo y macro sistémico, Berry et al. (2019) destacan el bienestar mental y el acceso a la educación (O’Leary et al., 2018) ya que mejoraron el empoderamiento y la eficacia de las familias.

De acuerdo a los recursos familiares resilientes se visibilizan cognitivamente en forma de creatividad, actividad e ingenio (Benavides, 2015). También se han reportado estrategias cognitivas de afrontamiento como olvidar y orar o recuperar identidades culturales (Chanmugam, 2015). Así mismo, se han experimentado cambios cognitivos, emocionales como el autoconcepto, el conocimiento de la 
VIF, el autocontrol y el optimismo (Crann \& Barata, 2016, 2019), la conciencia y comprensión, el entendimiento de las relaciones, la renovación y reconstrucción y resignificación de los eventos adversos (D’Amore, Martin, Wood, \& Brooks, 2018). También se reporta la recuperación y reconstrucción de la propia identidad, la sensación de libertad y salud, la aceptación y el perdón de sí mismo y el abusador, el reconocimiento del proceso a largo plazo y la posibilidad para superar el abuso (Flasch, Murray, \& Crowe, 2017). Adicionalmente se refieren fortalezas del carácter, gratitud, inteligencia social y curiosidad (Hellman \& Gwinn, 2017) esperanza (Hines, 2015), la autoestima (Matheson et al., 2015), la empatía y la tolerancia (Pérez-González, Guilera, Pereda, \& Jarne, 2017).

Se reconocieron recursos afectivos como, la búsqueda de recursos espaciales, físicos y corporales para protegerse (Alexander, Callaghan, Sixsmith, \& Fellin, 2015), la ecuanimidad (Bowen, 2017) y la entereza (Childress, Gioia, \& Campbell, 2018).

Comportamentalmente se reconocieron la: mediación, la solución de conflictos, la comunicación (Bain \& Durbach, 2018), el establecimiento de límites, las relaciones interpersonales positivas, la búsqueda de información y el apoyo por parte de la policía (Cherewick et al., 2015) la ayuda a otros, la solidaridad y el empoderamiento (Fellin et al., 2019; Goodman, et al, 2016), el emprendimiento (Green, Blattman, Jamison, \& Annan, 2015), la generación de redes de apoyo (Gregory, Williamson, \& Feder, 2017) y el desarrollo de habilidades prosociales (Howell, Thurston, Hasselle, Decker, \& Jamison, 2018).

En cuanto a los programas y estrategias de promoción de resiliencia, estos se caracterizaron por orientarse hacia el desarrollo de: Resistencia al control coercitivo a través del cuerpo y el espacio (Callaghan, Alexander, \& Fellin, 2016, Callaghan, Fellin, Mavrou, Alexander, \& Sixsmith, 2017) la recuperación de la experiencias y su resignificación (Callaghan, Fellin, \& Alexander, 2018; Grogan-Kaylor, Galano, Howell, Miller-Graff, \& Graham-Bermann, 2019), la expresión de sus emociones de manera profunda a través del drama (Dix, 2015).

Adicionalmente, se encontraron estrategias, como la regulación emocional bajo la supresión y la ree- valuación (Hong et al., 2018), al igual que la identificación de estrategias de control para intervenir en el proceso de lesión (Hartney \& Barnard, 2015), la intervención en atención primaria (Hegarty, Tarzia, Hooker, \& Taft, 2016), el enfoque integrado de cuerpo, mente y espíritu (Liao, 2019), el empoderamiento y el papel de la comunidad (Inman \& Rao, 2018; McDonald, Graham-Bermann, Maternick, Ascione, \& Williams, 2016), la comunicación madres e hijos para romper la VIF (Insetta et al., 2015), las intervenciones que construyen el capital social y la capacidad de recuperación de los jóvenes (Scorgie, et al, 2017) y la empatía frente a las historias de superhéroes como fuente de intervención en jóvenes (Weschenfelder, Fradkin, \& Yunes, 2018).

Frente a los hallazgos relacionados con la viF y de género, se observó un gran énfasis en que las depositarias de la VIF o de pareja son las mujeres, bien a nivel de la familia nuclear, extensa, o dentro de la violencia de pareja (Birdsall, Kirby, \& McManus, 2017; Morton \& Hohman, 2016; Sánchez \& Lopez-Zafra, 2019; Tsirigotis \& Łuczak, 2018; Ulloa, Hammett, Guzman, \& Hokoda, 2015) o en relación extendida con otros contextos como los hospitales (Arabaci, Dikec, Buyukbayram, Uzunoglu, \& Ozan, 2018). Aunque esté implícita la relación de la VIF con el género, sólo algunos estudios hacen patente esta relación (Asay et al., 2016).

También se observó la extensión del género, pero frente al ciclo evolutivo, tal como lo refiere Bain y Durbach (2018), las agrupaciones culturales ancestrales (Burrage, Gagnon, \& Graham-Bermann, 2018), mujeres refugiadas (Kim, Kim, \& Kim, 2016) y la extensión al abuso y violencia sexual (Murray, Moore Spencer, Stickl, \& Crowe, 2017).

Adicionalmente, la VIFG no solo se observa como un comportamiento actual, también se destaca el historial de maltrato, abuso, violencia en las familias y la sobrevivencia (Ben-David \& Jonson-Reid, 2017; LaBrenz, Dell, Fong, \& Liu, 2019; Meng, Fleury, Xiang, Li, \& D'arcy, 2018) o padres que han expuesto a sus hijos a violencia domestica (Scott, ThompsonWalsh, \& Nsiri, 2018).

Como complemento, también se destaca el marcado énfasis en los niños y niñas como lo resaltan, Beetham et al. (2019), Dubowitz et al. (2016), 
Elrofaie et al. (2016), Fong et al. (2019); Happer et al. (2017), McDonald, Corona et al. (2016) y McDonald, Graham-Bermann et al., (2016). Finalmente, en los jóvenes y adolescentes en la viFG (Ahmad, Ishtiaq, \& Mustafa, 2017; Romero, Hall, Cluver, \& Meinck, 2018) y adolescentes desde una visión de género (Namy et al., 2017; Noble, Ward, French, \& Falb, 2019).

\section{Discusión}

Frente a la cantidad de estudios publicados, estos pueden deberse a la prevalencia, el impacto positivo o negativo, a las posibilidades y beneficencia que representa estudiar o dirigirse al estudio de la VIFG y la resiliencia. Así pues, se visibiliza la perspectiva de género, lo cual sería consistente con la posición de la Organización Mundial de la Salud (OMs) (WHO, 2014) frente a la visibilización y abordaje de la VIF y de género (onu Mujeres, 2017).

Llama la atención que en Sur y Centroamérica no se reportan estudios, salvo el de Weschenfelder et al. (2018) y más cuando las refieren una altísima tasa de VIFG como lo refiere la United Nations for Development Program (UNDP) (2017). La Organización Mundial de la Salud, estima que el $30 \%$ de las mujeres que tienen o han tenido un compañero íntimo han experimentado violencia de parte de este, tanto física como sexual. La prevalencia fue mayor en África, el Mediterráneo Oriental y Asia Sudoriental (cerca del 37\%), y en las Américas (30\%) (oMs, 2014).

Si bien, el foco no es está dirigido a la resiliencia, si es preocupante que no se haga énfasis en la resiliencia y menos desde una visión de género lo que haría precisamente que se siga invisibilizando la incidencia de esta en la VIF, y obviamente la evitación de intervenciones positivas y generativas.

Considerando las publicaciones que difunden el conocimiento relacionado con los fenómenos de interés, se destacan que en su totalidad se relacionan con el fenómeno de la violencia intrafamiliar, de pareja, contra los niños y adolescentes. Es decir, responde a literatura especializada, y en menor medida de carácter general, de la misma forma resaltamos cómo la resiliencia empieza a cobrar relevancia como alternativa de investigación, reflexión y revisión en relación con la violencia y particularmente en revistas de un factor de impacto representativo ya que fluctúan entre el primer y segundo cuartil e índice h entre 129 y 14 (Tabla 11)

Tabla 11.

Clasificación siguiendo el Índice $\mathrm{H}$ y el cuartil de las revistas (Scopus) que difunden la relación de la VIFG y la resiliencia

\begin{tabular}{|c|c|c|}
\hline Journals & Cuartil & Índice $\mathrm{H}$ \\
\hline $\begin{array}{l}\text { Journal of Interpersonal } \\
\text { Violence }\end{array}$ & Q1 & 93 \\
\hline Child Abuse \& Neglect & Q1 & 129 \\
\hline Journal of Family Violence & Q2 & 69 \\
\hline $\begin{array}{l}\text { Child and Adolescent Social } \\
\text { Work Journal }\end{array}$ & Q2 & 38 \\
\hline $\begin{array}{l}\text { Children and youth services } \\
\text { review }\end{array}$ & Q1 & 77 \\
\hline $\begin{array}{l}\text { Journal of Child \& Adoles- } \\
\text { cent Trauma }\end{array}$ & Q2 & 14 \\
\hline Trauma, Violence, \& Abuse & Q1 & 70 \\
\hline $\begin{array}{l}\text { Aggression and Violent } \\
\text { Behavior }\end{array}$ & Q1 & 90 \\
\hline
\end{tabular}

Al respecto de los participantes o poblaciones de interés para los investigadores, aclaramos que, considerando que la búsqueda y problema de investigación se orientó hacia la violencia intrafamiliar y el género, no se observó que las publicaciones, excepto un caso Asay, et al. (2016), hicieran referencia de forma explícita a la violencia intrafamiliar y de género, sino que se infirió esta relación y se subrayó que la depositaria de la violencia es la mujer, las niñas y los niños, esto desde la casuística y no desde las implicaciones que aquí se refirieron previamente, dejando ver la invisibilización del género en la investigación e intervenciones relacionadas con la violencia intrafamiliar y la resiliencia.

Frente a los planteamientos de Jaramillo-Moreno \& Cuervo (2016), Jaramillo-Moreno (2017) y JaramilloMoreno \& Cuevas (2018), la resiliencia y la resiliencia familiar, deben ser consideradas como características o competencias emergentes de la identidad familiar, esto implica no verla desde una postura aislada sino relacional. Es decir, siguiendo a Cyrulnik (2010) se es resiliente en el marco de la relación y 
esta competencia funciona como dispositivo natural en la familia, el cual se presta para diferentes disciplinas, tal como se mencionó anteriormente, partiendo de las ciencias de la salud, la educación hasta las ciencias sociales y esta última en marcos, de la salud, lo organizacional, lo educativo o lo comunitario.

Por otro lado, comprendemos que la RF y los factores protectores, es decir aquellos que funcionan como dispositivos que atenúan, retrasan o blindan a las familia, frente a las adversidades, traumas o daños, son parte de la misma identidad resiliente, solo que el factor protector emergería como parte de la identidad y la historia familiar, comunitaria, educativa e institucional, lo cual deja entrever su lectura ecológica y en el marco de los sistemas amplios (Bronfenbrenner, 1987; Imer-Black, 2000). Esto, sin necesidad de ser respuesta ante la adversidad, pero que si funciona como base para el desarrollo y la emergencia de la resiliencia, siendo esta última la respuesta ante en este caso la VIFG.

\section{Conclusiones}

Como se destacó antes, frente a la invisibilización de la problemática, creemos que es un campo potencial, no solo ver el fenómeno de la VIFG sino también el análisis de la resiliencia y la resiliencia familiar, además del desarrollo continuo de programas orientados hacia estas.

Se destaca que es esencial diseñar estudios que consideren la resiliencia y la VIFG en América Latina y otros países, en donde estos fenómenos son de un alto impacto negativo, pues para Buvinie, et al. (1999) América Latina y obviamente Colombia son los contextos socio demográficos más violentos contra la mujer y consecuentemente la familia y que se beneficiarian de estas iniciativas investigativas e interventivas.

Es interesante cómo, frente a lo amplio de la literatura, se observa que la VIFG y la resiliencia, si bien se concentra en la literatura especializada, tiene una tendencia a elevarse en otras áreas del conocimiento que comprenden la Medicina, Psiquiatría,
Pediatría, Trabajo Social, Psicología y Educación lo cual coincide con la posición interdisciplinar de la resiliencia, la familia y el género.

Al respecto de los tipos de estudios se resalta cómo en el estudio de la resiliencia se tiene una marcada tendencia a los estudios cualitativos y su objetivo de comprender y descripción de fenómenos humanos, desde visiones complejas, sistémicas, interpretativas, culturales y transformadoras. Por otro lado, también se muestra cómo desde los estudios cuantitativos, el propósito obedecería a la sistematización de experiencias, protocolos o guías de intervención que promuevan la emergencia de la resiliencia ante eventos de adversidad, como de fenómenos de altísimo impacto negativo como la vIFG.

Con respecto al género y su relación directa y explícita, aún se observa que la investigación al respecto de la vif se desvincula, o al menos no explora la relación con la categoria del género; y además, la forma en que se suma a la diferenciación de los tipos de violencia en el marco de la familia. Esto, en tanto la violencia intrafamiliar, de pareja, infantil, e incluso el fenómeno del maltrato, dentro de la lectura sistémica y ecológica conforman un mismo fenómeno, es decir la familia, y que el ejercicio violento, así tenga un contexto "privado" o "secreto" afectará la totalidad del sistema familiar.

Con respecto a lo anterior, esta continuidad termina alimentando la invisibilización de la incidencia y justificación del género en la violencia, independientemente si se da dentro del subsistema conyugal o dentro del marco amplio del sistema familiar, que trasciende hacia la familia extensa y las generaciones familiares.

Considerando que la resiliencia familiar es la respuesta o, en otras palabras, una competencia emergente, relacional y dialéctica de la familia ante la VIFG, debe incluirse en su abordaje, conceptualización e interpretación el enfoque ecosistémico y de sistemas amplios. Esto, debido a que no se puede restringir su interpretación aisladamente al individuo o la familia nuclear, sino que es una competencia interdependiente a otros niveles, como por ejemplo, el nivel comunitario, social, nacional y global. 


\section{Referencias}

Ahmad, S., Ishtiaq, S. M., \& Mustafa, M. (2017). The role of socio-economic status in adoption of coping strategies among adolescents against domestic violence. Journal of Interpersonal Violence, 32(18), 2862-2881. https://doi. org/10.1177/0886260516635321

Alexander, J. H., Callaghan, J. E., Sixsmith, J., \& Fellin, L. (2015). Children's corporeal agency and use of space in situations of domestic violence. Play, Recreation, Health and Well Being, 9, 1-21. https://doi.org/10.1007/978-981-4585-51-4_23

Arabaci, L. B., Dikec, G., Buyukbayram, A., Uzunoglu, G., \& Ozan, E. (2018). Traumatic growth and psychological resilience status of female victims of violence inpatients in a district psychiatric hospital. Archives of Psychiatric Nursing, 32(4), 568-573. https:// doi.org/10.1016/j.apnu.2018.03.017

Arslan, G. (2016). Psychological maltreatment, emotional and behavioral problems in adolescents: The mediating role of resilience and selfesteem. Child Abuse \& Neglect, 52, 200-209. https://doi.org/10.1016/j.chiabu.2015.09.010

Asay, S. M., DeFrain, J., Metzger, M., \& Moyer, B. (2016). Implementing a strengths-based approach to intimate partner violence worldwide. Journal of Family Violence, 31(3), 349-360. https://doi.org/10.1007/s10896-015-9770-2

Bain, K., \& Durbach, C. (2018). Adaptation, Resilience, and Secure Attachment States of Mind in Young South African Female Students Exposed to Intimate Partner Violence in Childhood. Journal of Interpersonal Violence. https://doi. org/10.1177/0886260518759652

Beetham, T., Gabriel, L., \& James, H. (2019). Young children's narrations of relational recovery: A school-based group for children who have experienced domestic violence. Violence, 34, 565575. https://doi.org/10.1007/s10896-018-0028-7

Benavides, L. E. (2015). Protective factors in children and adolescents exposed to intimate partner violence: An empirical research review. Child and Adolescent Social Work Journal, 32(2), 93107. https://doi.org/10.1007/s10560-014-0339-3

Ben-David, V. \& Jonson-Reid, M. (2017). Resilience among adult survivors of childhood neglect: A missing piece in the resilience literature. Children and Youth Services Review, 78, 93-103. https://doi.org/10.1016/j. childyouth.2017.05.014

Berry, V., Wilkinson, K., Farr, N., \& Stimson, A. (2019). Assessing the feasibility of a parent life coaching intervention to support parents and children who have experienced domestic violence and abuse. Journal of Family Violence, 34, 493-506 https://doi.org/10.1007/ s10896-019-00042-6

Birdsall, N., Kirby, S., \& McManus, M. (2017). Police-victim engagement in building a victim empowerment approach to intimate partner violence cases. Police Practice and Research, 18(1), 75-86. https://doi.org/10.10 80/15614263.2016.1230061

Bowen, E. (2015). The impact of intimate partner violence on preschool children's peer problems: An analysis of risk and protective factors. Child Abuse \& Neglect, 50, 141-150. https://doi.org/10.1016/j.chiabu.2015.09.005

Bowen, E. (2017). Conduct disorder symptoms in pre-school children exposed to intimate partner violence: Gender differences in risk and resilience. Journal of Child \& Adolescent Trauma, 10(2), 97-107. https://doi.org/10.1007/ s40653-017-0148-x

Brino, Rachel Faria, \& Souza, Mayra Aparecida de Oliveira. (2016). Conceptions of Intrafamily Violence in the Educational Sector. Educação \& Realidade, 41(4), 1251-1273. https://dx.doi. org/10.1590/2175-623653298

Bronfenbrenner, U. (1987). La ecología del desarrollo humano. Ibérica: Paidós.

Burnette, C. E. (2018). Family and cultural protective factors as the bedrock of resilience and 
growth for indigenous women who have experienced violence. Journal of Family Social Work, 21(1), 45-62. https://doi.org/10.1080/1 0522158.2017.1402532

Burrage, R. L., Gagnon, M. \& Graham-Bermann, S. A. (2018). Trauma history and social support among American Indian/Alaska native and nonnative survivors of intimate partner violence. Journal of Interpersonal Violence. https:// doi.org/10.1177/08862 60518772103

Buvinie, M., Morrsion, A., \& Shifter, M. (1999). La Violencia en América Latina y el Caribe: Un Marco de Referencia para la Acción. Washington: Banco Interamericano de Desarrollo. Recuperado de https://www. cepal.org/mujer/noticias/paginas/3/27453/ BID.los\%20costos\%20de\%20la\%20violencia.pdf

Callaghan, J. E., Alexander, J. H., \& Fellin, L. C. (2016). Children's embodied experience of living with domestic violence: "I'd go into my panic, and shake, really bad”. Subjectivity, 9, 399-419 (2016). https:// doi.org/10.1057/s41286-016-0011-9

Callaghan, J. E. M., Fellin, L. C., Mavrou, S., Alexander, J., \& Sixsmith, J. (2017). The management of disclosure in children's accounts of domestic violence: Practices of telling and not telling. Journal of Child and Family Studies, 26(12), 3370-3387. https://doi. org/10.1007/s10826-017-0832-3

Callaghan, J. E., Fellin, L. C., \& Alexander, J. H. (2018). Promoting resilience and agency in children and young people who have experienced domestic violence and abuse: The "MPOWER" intervention. Journal of Family Violence, 34, $521-537$ (2019). https://doi.org/10.1007/ s10896-018-0025-x, 1-17.

Cameranesi, M. \& Piotrowski, C. C. (2018). Selfesteem in children exposed to intimate partner violence: A critical review of the role of sibling relationships and agenda for future research. Journal of Child \& Adolescent Trauma, 11(3), 339-351. https://doi.org/10.1007 /s40653-017-0180-x
Cassé, J. F., Finkenauer, C., Oosterman, M., van der Geest, V. R., \& Schuengel, C. (2018). Family conflict and resilience in parenting self-efficacy among high-risk mothers. Journal of Interpersonal Violence, 33(6), 1008-1029. https://doi.org/10.1177/ 0886260515614280.

Chanmugam, A. (2015). Young adolescents' situational coping during adult intimate partner violence. Child \& Youth Services, 36(2), 98-123. https://doi.org/10.1080/0145935X.2014.990627

Cherewick, M., Kohli, A., Remy, M. M., Murhula, C. M., Kurhorhwa, A. K. B., Kindja, G. M. (2015). Coping among trauma-affected youth: A qualitative study. Conflict and Health, 9(1), 9-35. https://doi.org/10.1186/s13031-015-0062-5

Childress, S., Gioia, D., \& Campbell, J. C. (2018). Women's strategies for coping with the impacts of domestic violence in Kyrgyzstan: A grounded theory study. Social Work in Health Care, 57(3), 164-189. https://doi.org/10.1080/0 0981389.2017.1412379

Crann, S. E., \& Barata, P. C. (2016). The experience of resilience for adult female survivors of intimate partner violence: A phenomenological inquiry. Violence Against Women, 22(7), 853-875. https://doi.org/10.1177/1077801215612598.

Crann, S. E., \& Barata, P. C. (2019). "We can be oppressed but that does not mean we cannot fight oppression": Narratives of resilience and advocacy from survivors of intimate partner violence. Journal of Interpersonal Violence. https://doi.org/10.1177/0886260519848779

Cuevas, C. (2017). Orientaciones sexuales e identidades de género diversas: comprensiones complejas, ecológicas y sistémicas en el currículo de un programa de Psicología. En Editores R., Zapata, R., Dalouh, V.,Cala, A, González. Educación, Salud y Tic en contextos multiculturales: nuevos espacios de intervención. Almería: Editorial Universidad de Almería.

Cyrulnik, B. (2010). La Resiliencia. Conceptualización de la resiliencia. El arte de resurgir a la vida. 1. Recuperado de http://dspace.ups.edu.ec/bitstream/1234567 89/2801/1/UPS- QT01138.pdf 
D’Amore, C., Martin, S. L., Wood, K., \& Brooks, C. (2018). Themes of healing and posttraumatic growth in women survivors' narratives of intimate partner violence. Journal of Interpersonal Violence. https://doi. org/10.1177/0886260518767909.

Dhunna, S., Lawton, B., \& Cram, F. (2018). An affront to her mana: Young māori mothers' experiences of intimate partner violence. Journal of Interpersonal Violence, https://doi. org $/ 10.1177 / 0886260518815712$

Dix, A. (2015). Telling stories: Dramatherapy and theatre in education with boys who have experienced parental domestic violence. Dramatherapy, 37(1), 15-27. https://doi.org/1 0.1080/02630672.2015.1055778

Dubowitz, H., Thompson, R., Proctor, L., Metzger, R., Black, M. M., ... Magder, L. (2016). Adversity, maltreatment, and resilience in young children. Academic Pediatrics, 16(3), 233-239. https:// doi.org/10.1016/j.acap.2015.12.005.

Elrofaie, A., Stewart, S., \& Schwean, V. (2016). Resilience amid maltreatment and domestic violence: Mediating impacts of risk and protective factors on child psychopathology. Personality and Individual Differences, 100(101), 476.

Faria Brino, R. \& de Oliveira Souza, M. A. (2016). Conceptions of Intrafamily Violence in the Educational Sector. Educação e Realidade, 41(4), 1251-1272. Recuperado de: https:// doi-org.crai-ustadigital.usantotomas.edu. co/10.1590/2175-623653298

Fellin, L. C., Callaghan, J. E., Alexander, J. H., Harrison-Breed, C., Mavrou, S., \& Papathanasiou, M. (2019). Empowering young people who experienced domestic violence and abuse: The development of a group therapy intervention. Clinical Child Psychology and Psychiatry, 24(1), 170-189.

Flasch, P., Murray, C. E., \& Crowe, A. (2017). Overcoming abuse: A phenomenological investigation of the journey to recovery from past intimate partner violence. Journal of
Interpersonal Violence, 32(22), 3373-3401. https://doi.org/10.1177/0886260515599161.

Flick, U. (2004) Introducción a la Investigación cualitativa. Madrid: Morata

Fong, V. C., Hawes, D., \& Allen, J. L. (2019). A systematic review of risk and protective factors for externalizing problems in children exposed to intimate partner violence. Trauma, Violence, \& Abuse, 20(2), 149-167. https://doi. org/10.1177/1524838017692383

Ghafournia, N. (2017). Muslim women and domestic violence: Developing a framework for social work practice. Journal of Religion \& Spirituality in Social Work: Social Thought, 36(1-2), 146-163. https://doi.org/10.1080/15426432.2017.1313150

Goodman, L. A., Thomas, K., Cattaneo, L. B., Heimel, D., Woulfe, J., \& Chong, S. K. (2016). Survivor-defined practice in domestic violence work: Measure development and preliminary evidence of link to empowerment. Journal of Interpersonal Violence, 31(1), 163-185. https:// doi.org/10.1177/0886260514555131

Grant, M. J. \& Booth, A. (2009). A typology of reviews: An analysis of 14 review types and associated methodologies. Health Information \& Libraries Journal, 26(2), 91-108.

Green, E. P., Blattman, C., Jamison, J., \& Annan, J. (2015). Women's entrepreneurship and intimate partner violence: A cluster randomized trial of microenterprise assistance and partner participation in post-conflict Uganda (SSM-D-14-01580R1). Social Science \& Medicine, 133, 177-188. https://doi.org/10.1016/j.socscimed.2015.03.042

Gregory, A. C., Williamson, E., \& Feder, G. (2017). The impact on informal supporters of domestic violence survivors: A systematic literature review. Trauma, Violence, \& Abuse, 18(5), 562580. https://doi.org/10.1177/1524838016641919

Grogan-Kaylor, A., Galano, M. M., Howell, K. H., Miller-Graff, L., \& Graham-Bermann, S. A. (2019). Reductions in parental use of corporal 
punishment on pre-school children following participation in the moms' empowerment program. Journal of Interpersonal Violence, 34(8), 1563-1582. https://doi. org/10.1177/0886260516651627

Happer, K., Brown, E. J., \& Sharma-Patel, K. (2017). Children's resilience and trauma-specific cognitive behavioral therapy: Comparing resilience as an outcome, a trait, and a process. Child Abuse \& Neglect, 73, 30-41. https://doi. org/10.1016/j.chiabu.2017.09.021

Hartney, E. \& Barnard, D. K. (2015). A framework for the prevention and mitigation of injury from family violence in children of parents with mental illness and substance use problems. Aggression and Violent Behavior, 25, 354-362.

Hashemi, S. \& Akbari, A. (2017). Causal model for family communication patterns, resilience and control of anger with tendency to violence. Journal of Psychology, 21(3), 284-299.

Hegarty, K., Tarzia, L., Hooker, L., \& Taft, A. (2016). Interventions to support recovery after domestic and sexual violence in primary care. International Review of Psychiatry, 28(5), 519-532.

Hellman, C. M. \& Gwinn, C. (2017). Camp hope as an intervention for children exposed to domestic violence: A program evaluation of hope, and strength of character. Child and Adolescent Social Work Journal, 34(3), 269-276. https:// doi.org/10.1007/s10560-016-0460-6

Hernández, A. \& Bravo, F. (2004). Vínculos, ecología y redes. Hallazgos, 1(1), 111-129.

Hines, L. (2015). Children's coping with family violence: Policy and service recommendations. Child and Adolescent Social Work Journal, 32(2), 109119. https://doi.org/10.1007/s10560-014-0333-9

Hong, F., Tarullo, A. R., Mercurio, A. E., Liu, S., Cai, Q., \& Malley-Morrison, K. (2018). Childhood maltreatment and perceived stress in young adults: The role of emotion regulation strategies, self-efficacy, and resilience. Child Abuse \&
Neglect, 86, 136-146. https://doi.org/10.1016/j. chiabu.2018.09.014.

Howell, K. H., Thurston, I. B., Hasselle, A. J., Decker, K., \& Jamison, L. E. (2018). Systemic factors associated with prosocial skills and maladaptive functioning in youth exposed to intimate partner violence. Journal of Interpersonal Violence, https://doi.org/10.1177/0886260518766420

Inman, A. G. \& Rao, K. (2018). Asian Indian women: Domestic violence, mental health, and sites of resilience. Women \& Therapy, 41(1-2), 83-96.

Instituto Colombiano de Bienestar Familiar-ICBF (2006). Lineamientos técnicos, administrativomisionales para la inclusión y la atención de familias en los programas y servicios del ICFB. Bogotá: ICBF

Instituto Nacional de Medicina Legal y Ciencias Forenses, (2018). Boletín estadístico mensual, octubre de 2018. Bogotá: Instituto Nacional de Medicina Legal y Ciencias Forenses. Recuperado de: http://www.medicinalegal. gov.co/documents/ 20143/217010/ Diciembre 2018_Ajustado.pdf/6142c980-e4e3-c2df-f232b3547bac4a29

Imer-Black, E. (2000) Familias y sistemas amplios, el terapeuta familiar en el laberinto. Buenos Aires: Amorrortu.

Insetta, E. R., Akers, A. Y., Miller, E., Yonas, M. A., Burke, J. G., ... Chang, J. C. (2015). Intimate partner violence victims as mothers: Their messages and strategies for communicating with children to break the cycle of violence. Journal of Interpersonal Violence, 30(4), 703724. https://doi.org/10.1177/0886260514535264

Jahoda, M. (1958). Current Concepts of Positive Mental Health. Nueva York: Basic Books.

Jaramillo-Moreno, R. \& Cuervo, J. (2016) El resurgir de las familias militares frente a la adversidad. Exploración de los recursos resilientes en familias frente a la privación de la libertad por acciones del servicio. Bogotá: Universidad de la Sabana, Conferencia dentro de marco del VIII 
Congreso Internacional de la Familia, Mi casa, Territorio de Paz.

Jaramillo-Moreno, R. (2017) Resiliencia familiar, comprensión, campos de aplicación, aportes y desafío. Diversitas: Perspectivas en Psicología, 13(2), 255-266.

Jaramillo-Moreno, R. \& Cuevas, C. (2018). Informe Final del Proyecto: Resiliencia en víctimas de Violencia intrafamiliar y de Género Fase 1. Bogotá: Universidad Santo Tomás de Aquino.

Kassis, W., Artz, S., Scambor, C., Scambor, E., \& Moldenhauer, S. (2013). Finding the way out: A non-dichotomous understanding of violence and depression resilience of adolescents who are exposed to family violence. Child Abuse \& Neglect, 37(2-3), 181-199. https://doi. org/10.1016/j.chiabu.2012.11.001.

Kim, H. J., Kim, J. Y., \& Kim, D. G. (2016). “Thank you, sorry, love" (TSL) therapy with North Korean refugee women: A pilot study. Research on Social Work Practice, 26(7), 816-824. https:// doi.org/10.1177/1049731515570137

Kotliarenco, M. \& Gomez, E. (2010) Resiliencia Familiar: un enfoque de investigación e intervención con familias multiproblemáticas. Revista de Psicología, 9(2), 103 - 132.

LaBrenz, C. A., Dell, P. J., Fong, R., \& Liu, V. (2019). Happily ever after? life satisfaction after childhood exposure to violence. Journal of Interpersonal Violence. https://doi. org $/ 10.1177 / 088626051882070$

Liao, M. (2019). Integrating body-mind-spirit services used by women experiencing intimate partner violence: A case study in Taiwan. International Social Work, 62(2), 549-566. https://doi.org/10.1177/0020872817732378

Lluch, M. (2002). Evaluación empírica de un modelo conceptual de salud mental positiva. Salud mental, 25(4), 42-55.

Matheson, F. I., Daoud, N., Hamilton-Wright, S., Borenstein, H., Pedersen, C., \& O'Campo, P. (2015). Where did she go? the transformation of self-esteem, self-identity, and mental wellbeing among women who have experienced intimate partner violence. Women's Health Issues, 25(5), 561-569. https://doi.org/10.1016/j. whi.2015.04.006

McDonald, S. E., Corona, R., Maternick, A., Ascione, F. R., Williams, J. H., \& Graham-Bermann, S. A. (2016). Children's exposure to intimate partner violence and their social, school, and activities competence: Latent profiles and correlates. Journal of Family Violence, 31(7), 849-864. https://doi.org/10.1007/s10896-016-9846-7

McDonald, S. E., Graham-Bermann, S. A., Maternick, A., Ascione, F. R., \& Williams, J. H. (2016). Patterns of adjustment among children exposed to intimate partner violence: A person-centered approach. Journal of Child \& Adolescent Trauma, 9(2), 137-152. https://doi.org/10.1007/ s40653-016-0079-y

Meng, X., Fleury, M., Xiang, Y., Li, M., \& D’arcy, C. (2018). Resilience and protective factors among people with a history of child maltreatment: A systematic review. Social Psychiatry and Psychiatric Epidemiology, 53(5), 453-475. https://doi.org/10.1007/s00127-018-1485-2

Morton, S. \& Hohman, M. (2016). “That's the weight of knowing": Practitioner skills and impact when delivering psychoeducational group work for women who have experienced IPV. Social Work with Groups, 39(4), 277-291. https://doi. org/10.1080/01609513.2015.1052915

Mrazek, P. J. \& Mrazek, D. (1987). Resilience in Child Maltreatment Victims: A Conceptual Exploration. Child Abuse and Neglect, 11(3), 339-362. https://doi. org/10.1016/0145-2134(87)90009-3

Murray, C. E., Moore Spencer, K., Stickl, J., \& Crowe, A. (2017). See the triumph healing arts workshops for survivors of intimate partner violence and sexual assault. Journal of Creativity in Mental Health, 12(2), 192-202. https://doi.org $/ 10.1080 / 15401383.2016 .1238791$

Namy, S., Carlson, C., Pala, A. N., Faris, D., Knight, L., Naker, D. (2017). Gender, violence and re- 
silience among Ugandan adolescents. Child Abuse \& Neglect, 70, 303-314. https://doi. org/10.1016/j.chiabu.2017.06.015

Noble, E., Ward, L., French, S., \& Falb, K. (2019). State of the evidence: A systematic review of approaches to reduce gender-based violence and support the empowerment of adolescent girls in humanitarian settings. Trauma, Violence, \& Abuse, 20(3), 428-434. https://doi. org/10.1177/1524838017699601

O'Leary, P., Cameron, C. M., Lakhani, A., Osborne, J. M., de Souza, L., Majidi, S. (2018). Violence against children in Afghanistan: Concerns and opportunities for positive change. Child Abuse \& Neglect, 76, 95-105. https://doi.org/10.1016/j. chiabu.2017.10.010

ONU Mujeres. (2017). Hechos y cifras: Acabar con la violencia contra mujeres y niñas. Recuperado de http://www.unwomen.org/es/ what-we-do/ending-violence-against-women/ facts-and-figures

Pérez-González, A., Guilera, G., Pereda, N., \& Jarne, A. (2017). Protective factors promoting resilience in the relation between child sexual victimization and internalizing and externalizing symptoms. Child Abuse \& Neglect, 72, 393-403. https://doi.org/10.1016/j.chiabu.2017.09.006

Romero, R. H., Hall, J., Cluver, L., \& Meinck, F. (2018). Can supportive parenting protect against school delay amongst violence-exposed adolescents in South Africa? Child Abuse \& Neglect, 78, 31-45. https://doi.org/10.1016/j. chiabu.2017.09.025

Roy, M. (2018) The impact of resilience and recovery among the survivors of domestic violence: A perspective study of women in the Indian context. Indian Journal of Health \& Wellbeing, 5(12), 1487-1491. https://doi.org/10.1007/ s11126-017-9529-4

Rutter, M. (1993). Resilience: Some conceptual considerations. Journal of Adolescent Health, 14(8), 626-631.
Sánchez, M. F. \& Lopez-Zafra, E. (2019). The voices that should be heard: A qualitative and content analysis to explore resilience and psychological health in victims of intimate partner violence against women (IPVAW). Paper presented at the Women's Studies International Forum, 72, 80-86.

Schaefer, L. M., Howell, K. H., Thurston, I. B., Kaufman, C. C., \& Hasselle, A. J. (2018). Protective factors associated with fewer generalized anxiety disorder symptoms among women exposed to intimate partner violence. Journal of Interpersonal Violence, https:// doi.org/10.1177/0886260518817027.

Schofield, G., Biggart, L., Ward, E., \& Larsson, B. (2015). Looked after children and offending: An exploration of risk, resilience and the role of social cognition. Children and Youth Services Review, 51, 125-133. https://doi.org/10.1016/ j.childyouth.2015.01.024

Scorgie, F., Baron, D., Stadler, J., Venables, E., Brahmbhatt, H., Delany-Moretlwe, S. (2017). From fear to resilience: Adolescents' experiences of violence in inner-city Johannesburg, South Africa. Bмc Public Health, 17(3), 441. https://doi.org/10.1186/s12889-017-4349-x

Scott, K. L., Thompson-Walsh, C., \& Nsiri, A. (2018). Parenting in fathers who have exposed their children to domestic violence: Identifying targets for change. International Journal on Child Maltreatment: Research, Policy and Practice, 1(1), 51-75. https://doi.org/10.1007/ s42448-018-0004-0

Sirikantraporn, S. J. (2017). Bicultural identity as a protective factor among southeast Asian American youth who have witnessed domestic violence. Transition and change in collectivist family life, 89, 97. https://doi. org/10.1007/978-3-319-50679-1_9

Smith, L. B., Lenz, A. S., \& Strohmer, D. (2017). Differential prediction of resilience among individuals with and without a history of abuse. 
Counseling and Values, 62(1), 106-122. https:// doi.org/10.1002/cvj.12052

Stephens, T. \& Aparicio, E. M. (2017). “It's just broken branches": Child welfare-affected mothers' dual experiences of insecurity and striving for resilience in the aftermath of complex trauma and familial substance abuse. Children and Youth Services Review, 73, 248-256. https:// doi.org/10.1016/j.childyouth.2016.11.035

Taylor, S. \& Bogdan, R. (1987) Introducción a los métodos cualitativos de Investigación. Barcelona: Paidós.

Tsirigotis, K. \& Łuczak, J. (2018). Resilience in women who experience domestic violence. Psychiatric Quarterly, 89(1), 201-211.

Ulloa, E. C., Hammett, J. F., Guzman, M. L., \& Hokoda, A. (2015). Psychological growth in relation to intimate partner violence: A review. Aggression and Violent Behavior, 25, 88-94. https://doi.org/10.1016/j.avb.2015.07.007

United Nations for Development Program (2017) Comparación de las políticas sobre violencia doméstica en América Latina: penalización, empoderamiento de víctimas y rehabilitación de agresores. UNDP. New York. EE. Uu. Recuperado de: https://www.undp.org/ content/dam/el_salvador/docs/womempow/ CuadernoGenero3_SP.pdf

Walsh, F. (2004) Resiliencia Familiar, estrategias para su fortalecimiento. Buenos Aires: Amorrortu.
Walsh, F. (2011). Facilitating family resilience: relational resources for positive youth development in conditions of adversity. The Social Ecology of Resilence, [CE1] 173-185. https://doi. org/10.1007/978-1-4614-0586-3_15

Walters, A. (2016). Stories of survival and resilience: An enquiry into what helps tamariki and rangatahi through whānau violence. New Zealand Journal of Psychology, 46 (3), 80-87.

Werner, E. E. \& Smith, R. S. (1982). Vulnerable but invincible: A longitudinal study of resilient children and youth. NuevaYork: McGraw Hill.

Weschenfelder, G. V., Fradkin, C., \& Yunes, M. A. M. (2018). Super-heróis como recursos para promoção de resiliência em crianças e adolescentes. Psicologia: Teoria e Pesquisa, 33(1).

World Health Organization (wHo). (2014) Global Status Report on Violence Prevention. wHo. Washington, EE. Uu. Recuperado de: https:// oig.cepal.org/sites/default/files/ informe_sobre_la_situacion_mundial_de_la_prevencion_ de_la_violencia.pdf

Wortham, T. (2014). Intimate Partner Violence: Building Resilience with Families and Children. Reclaiming Children \& Youth, 23(2), 58-61.

Yule, K., Houston, J., \& Grych, J. (2019). Resilience in Children exposed to Violence: A Metaanalysis of protective factors across ecological contexts. Clinical Child and Family Psychology Review. https://doi.org/10.1007 / s10567-019-00293-1 